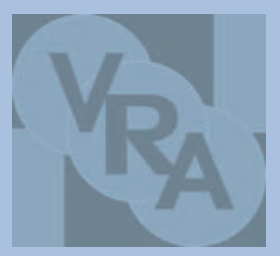

\title{
Einzelfallprüfungen, Prüfquoten und Strafzahlungen nach dem MDK-Reformgesetz in der Corona-Pandemie
}

Die Corona-Pandemie hatte auch einen Einfluss auf die MDK-Prüfungen. Viele der gesetzgeberischen Aktivitäten wurden zeitlich verschoben. So gilt nach der für 2020 reduzierten Prüfquote von 5\% für 2021 nun eine einheitliche Prüfquote von 12,5\% (Stand Januar 2021). Erst ab 2022 sollen dann quartalsbezogene, von den Quoten gekürzter Rechnungen abhängige krankenhausbezogene Prüfquoten und Strafzahlungen zum Einsatz kommen. Aus den Statistiken ${ }^{1}$ der Kostenträger kann bereits antizipiert werden, mit welchen Prüf- und Strafzahlungsquoten ein Krankenhaus rechnen muss. Die Strafzahlung beträgt minimal $300 €$ für jeden Fall mit Rechnungskürzung. Aufgrund der geringen Vergütungen für rheumatologische Fälle, dürften Strafzahlungen meist nur geringfügig höher ausfallen².

Eine Vereinbarung über die einzelfallbezogene Erörterung zwischen Krankenhaus und Krankenkasse bei Dissens über das MD-Gutachten, die Voraussetzung für eine nachfolgende gerichtliche Klärung des Sachverhaltes wird, ist der Deutschen Krankenhausgesellschaft und dem GKV-Spitzenverband 2020 nicht gelungen. Entsprechend ist erst im Laufe des Jahres 2021 mit einer neuen Prüfverfahrensvereinbarung (PrüfvV) und Umsetzungshinweisen zu dieser zu rechnen. Bis dahin bleiben auch Rechnungs-

$1 \quad$ www.gkv-spitzenverband.de/krankenversi cherung/krankenhaeuser/krankenhaeuser_ abrechnung/kh_pruefung_statistik/pruef quoten_und_statistik.jsp

2

Über die Höhe von $300 €$ hinaus wird die Strafzahlung auf maximal $10 \%$ des resultierenden Rechnungsbetrages begrenzt. D. h. erst bei einem resultierenden Rechnungsbetrag von $>3000 €(z$. B. bei der Abrechnung von ZE oder NUB für Biologika) entfalten die unterschiedlichen Sanktionsquoten ( $25 \% / 50 \%$ des Differenzbetrags zwischen Ursprungsrechnung und gekürzter Rechnung) in der Rheumatologie eine relevante Wirkung. korrekturen durch die Krankenhäuser ebenso wie Aufrechnungen durch die Krankenkassen wie im bisherigen Rahmen zulässig. Die Einführung des verpflichtenden Datenaustausches zwischen Krankenhaus und den Medizinischen Diensten wird sich ebenfalls weiter verzögern.

\section{Strukturprüfungen durch den Medizinischen Dienst (MD)}

Auch die Einführung der Strukturprüfungen wurde wegen der Corona-Pandemie um ein Jahr verschoben. Die Einhaltung der Strukturkriterien von OPS-Komplexkodes ist nun erst ab 2022 von den Krankenhäusern durch eine bestandene Strukturprüfung nachzuweisen. Die hierfür erforderliche Richtlinie ( $\$ 283$ SGB V) wird vom Medizinischen Dienst des Spitzenverbandes Bund der Krankenkassen erlassen werden. Für 2021 hat das BfArM (ehemals DIMDI) im OPS definiert, welche Mindestkriterien als Strukturkriterien anzusehen und daher in den Strukturprüfungen zu überprüfen sind. Für die rheumatologischen Komplexbehandlungen ist dies einzig das „Team unter fachärztlicher Behandlungsleitung“, wobei die Behandlungsleitung die fachlich-inhaltliche Verantwortung für die Versorgung der Patienten tragen muss. „Sie plant, koordiniert und überwacht die Leistungen und ärztlichen Tätigkeiten am Patienten. “ Es ist davon auszugehen, dass der MD, die Qualifikation und irgendeine Form der kontinuierlichen Sicherstellung der Behandlungsleitung prüfen wollen wird. Gegebenenfalls wird der MD auch Vorhandensein, Zusammensetzung und Qualifikation des Teams prüfen wollen. Ob die Prüftiefe (z. B. tägliche Anwesenheiten) bereits in der Richtlinie geregelt wird oder dem einzelnen MD und/oder seinen Gutachtern überlassen bleibt, ist noch nicht bekannt. Die Strukturmerkmale der rheumatologischen Komplexbehandlungen waren und sind - im Gegen- satz zu beispielsweise frührehabilitativen Komplexbehandlungen - unabhängig von der Corona-Pandemie vollständig einzuhalten und nachzuweisen.

Wichtig ist, dass der MD nicht von sich aus die Strukturkriterien prüft, sondern Krankenhäuser ihn dazu explizit auffordern müssen. Rheumatologischen Kliniken sei deshalb dringlich angeraten, nach Veröffentlichung der Richtlinie frühzeitig beim MD eine Strukturprüfung anzufordern, damit sie eine eventuell verspätete Prüfung nicht selbst zu verantworten haben. Erfahrungen aus bisherigen Strukturprüfungen des MDK zeigen trotz guter Vorbereitung der Krankenhäuser hohe Durchfallquoten bei den ersten Prüfungen. Die damit verbundenen, potenziell dramatischen wirtschaftlichen Risiken sollten rheumatologische Kliniken nicht übersehen!

\section{Schlichtungsausschuss Bund}

Der Schlichtungsausschuss Bund hat 2020 seine Arbeit aufgenommen und sich in den Konstellationen zur Kodierung positioniert, in denen es einen Dissens zwischen der Sozialmedizinischen Expertengruppe 4 (SEG) des MDK und dem Fachausschuss für ordnungsgemäße Kodierung und Abrechnung (FoKA) der Deutschen Gesellschaft für Medizincontrolling gab. Soweit möglich flossen die den Entscheidungen zu entnehmenden Interpretationen der zugrundeliegenden Klassifizierung- und Kodierrichtlinien in den aktuellen Kodierleitfaden Rheumatologie 2021 ein. Im Laufe des Jahres 2021 sind weitere Entscheidungen zu erwarten. Da der Kodierleitfaden Rheumatologie nur jährlich überarbeitet wird, können diese Entscheidungen erst in die Revision für 2022 eingepflegt werden. Zu Beginn des Jahres wurde die Geschäftsordnung veröffentlicht und das allgemeine Antragsverfahrens eröffnet (www.g-drg.de/Schlichtungsausschuss_ 
nach_19_KHG). Antragsberichtigt ist auch jedes einzelne Krankenhaus. Es ist jedoch zu beachten, dass der Schlichtungsausschuss Bund nur über Kodier- und Abrechnungsfragen von „grundsätzliche Bedeutung " 3 entscheidet und die Beantragung kostenpflichtig ist (1190€).

\section{Pflege und Weiterentwicklung der Klassifikationssysteme}

Das Deutsche Institut für Medizinische Dokumentation und Information (DIMDI), das bislang die Klassifikationssysteme ICD-10GM und OPS pflegte, wurde 2020 formal in das Bundesinstitut für Arzneimittel und Medizinprodukte (BfArM) eingegliedert. Die Aufgaben, der Status als Behörde im Ressort des Bundesministeriums und der Dienstsitz Köln sind aber erhalten geblieben.

Da die Corona-Pandemie die Krankenhäuser 2021 noch begleiten und auch Rheumakliniken mit betreffen wird, ist die spezifische Kodierung, die derzeit noch im Wesentlichen auf temporären Kodes (U07.-, U08.9, 109.9!, U10.9 und U99.0!) basiert, zu beachten $^{4}$. Kurzfristige Anpassungen durch die WHO und das BfArM sind möglich. Die Ko-

3 Nähere Definition in $\S 10$ der Vereinbarung über die Bildung des Schlichtungsausschusses nach § 19 KHG

4

Offizielle Kodierhinweise finden sich unter: www.dimdi.de/dynamic/de/klassifikationen/ icd/icd-10-gm/ dierung ist sowohl für den Corona-Mehrkostenzuschlag als auch für das Zusatzentgelt für Testungen auf das Coronavirus relevant.

Ein aktueller Gesetzentwurf (DVPMG ${ }^{5}$ ) sieht vor, dass ab 2022 zur Spezifizierung bei Vorliegen einer „seltenen Erkrankung“ in der stationären Versorgung neben einem ICD-Kode auch noch zusätzlich eine Orphanet-Kennnummer anhand der Alpha-ID-SE zu verschlüsseln ist. Dies wird die Kodierung in der Rheumatologie komplexer werden lassen. So können beispielsweise für den systemischen Lupus erythematodes je nach Art 4 unterschiedliche Alpha-ID-SE vergeben werden (autosomal-rezessiver systemischer Lupus erythematodes, disseminierter/systemischer Lupus erythematodes, systemischer Lupus erythematodes des Kindesalters und medikamenteninduzierter Lupus erythematodes). Sollte das Gesetz wie geplant umgesetzt werden, wird der VRA den Kodierleitfaden für 2022 diesbezüglich rechtzeitig ergänzen.

Auch wenn von vielen die Zukunft des G-DRG-Systems in Frage gestellt wird, ist es nicht realistisch, dass die mit dem

5 Entwurf eines Gesetzes zur digitalen Modernisierung von Versorgung und Pflege (Digitale-Versorgung-und-Pflege-Modernisierungs-Gesetz - DVPMG), www. bundesgesundheitsministerium.de/ fileadmin/Dateien/3_Downloads/Gesetze_ und_Verordnungen/GuV/D/DVPMG_GEKabinett.pdf
G-DRG-System eingeführten Dokumentationspflichten wieder abgeschafft werden. Der VRA wird Sie daher weiterhin bei der korrekten Klassifizierung und Kodierung in der Rheumatologie mit dem Kodierleitfaden unterstützen.

Dr. med. Wolfgang Fiori, DRG-ResearchGroup, Roeder \& Partner - Ärzte Part G, Am Dorn 10, 48308 Senden

Prof. Dr. med. Heinz-Jürgen Lakomek

Prof. Dr. med. Norbert Roeder, DRG-Research-Group, Roder \& Partner - Ärzte Part G, Am Dorn 10, 48308 Senden

\section{KONTAKTADRESSE}

Verband Rheumatologischer Akutkliniken e. V.

Geschäftsstelle

Schumannstr. 18, 10117 Berlin

Tel./Fax: 030/20 62 98-79/-82

E-Mail: gf@vraev.de

Internet: www.vraev.de

\section{IMPRESSUM}

\section{Verantwortlich für den Inhalt}

Prof. Dr. Heinz-Jürgen Lakomek, Direktor, Universitätsklinik für Geriatrie, Johannes Wesling Klinikum Minden 